\title{
Dynamical conductance through InAs/GaSb/InAs and InAs/AISb/GaSb/AISb/InAs structures
}

\author{
Pui Wai Ma ${ }^{1}$ and Jian Wang ${ }^{1,2, *}$ \\ ${ }^{1}$ Department of Physics, The University of Hong Kong, Pokfulam Road, Hong Kong, China \\ ${ }^{2}$ Key Laboratory of Materials Physics, Institute of Solid State Physics, Chinese Academy of Sciences, Hefei, Anhui, \\ People's Republic of China \\ (Received 17 April 2003; revised manuscript received 30 September 2003; published 10 March 2004)
}

\begin{abstract}
Using the general current-conserving theory developed by Buttiker for ac transport, the dynamical conductance of InAs/GaSb/InAs and InAs/AlSb/GaSb/AlSb/InAs structures are calculated within the two-band $\mathrm{k} \cdot \mathrm{p}$ model. In these interband systems, there is an energy window $\Delta E$ within which the transmission coefficient is nonzero. When the frequency of the external bias is comparable to this energy window, the real part of the dynamical conductance shows a series of plateaus that are well correlated with the profile of the transmission coefficient. The number and positions of the plateaus can be varied by changing the frequency. At frequencies much smaller than $\Delta E$, the phase of the dynamical conductance is well described by the emittance. As the frequency is increased, the capacitivelike and inductivelike behaviors observed at off-resonance and onresonance, respectively, are enhanced.
\end{abstract}

DOI: 10.1103/PhysRevB.69.125308

PACS number(s): 73.40.-c, 72.80.Ey, 73.63.-b

\section{INTRODUCTION}

The polytype heterostructures which consist of AlSb, $\mathrm{GaSb}$, and InAs were proposed by Esaki et al. ${ }^{1}$ in 1981. The special alignment of the conduction and valence bands (i.e., the 1.5-eV overlapping of the InAs conduction band and the $\mathrm{GaSb}$ valence band) as well as the coupling between them make the interband tunneling possible. Sweeny and $\mathrm{Xu}^{2}$ proposed resonant interband tunneling (RIT) diodes which incorporated resonant and interband tunneling in quantum wells, and resulted in high speed and low excess current. When a large forward bias is applied, a negative differential resistance (NDR) can be observed. Later on, Söderström et $a .^{3}$ proposed and demonstrated NDR and a high peak-tovalley $(\mathrm{P} / \mathrm{V})$ current ratio in the InAs/AlSb/GaSb/AlSb/InAs structure. Such a structure is considered as a RIT diode which is a combination of an Esaki diode and a doublebarrier resonant diode. On the theoretical side, Yang and $\mathrm{Xu}^{4}$ analyzed the dependence of the energy levels and lifetime of resonant states on the well width and barrier thickness of the leaky quantum well systems, which are $\mathrm{GaSb} / \mathrm{InAs} / \mathrm{GaSb}$, InAs/GaSb/InAs, GaSb/AlSb/InAs/AlSb/GaSb, and InAs/ $\mathrm{AlSb} / \mathrm{GaSb} / \mathrm{AlSb} / \mathrm{InAs}$. Using two-band $\mathrm{k} \cdot \mathrm{p}$ theory, the transmission properties of RIT in the polytype heterostructures based on InAs, AlSb, and GaSb are also analyzed. ${ }^{5,6}$ Nevertheless, a more realistic description of the RIT would be the eight-band $\mathrm{k} \cdot \mathrm{p}$ theory. ${ }^{7}$ This is because eight-band $\mathrm{k}$ - $\mathrm{p}$ theory also considers the heavy-hole and split-off bands, ${ }^{8}$ while the two-band $\mathrm{k} \cdot \mathrm{p}$ theory just considers the light-hole and conduction bands. Indeed, heavy-hole and split-off bands do contribute in the transmission process. ${ }^{9}$ However, if the wave vector is taken to be normal to the interfaces $\left(k_{\|}\right.$ $=0$ ), the heavy-hole and split-off bands are negligible, ${ }^{8,10}$ due to the symmetry of the Bloch functions. ${ }^{11}$ Davidovich et al. ${ }^{12}$ calculated the density of states and the currentvoltage $(I-V)$ characteristics of InAs/AlSb/GaSb/AlSb/InAs and $\mathrm{GaSb} / \mathrm{AlSb} / \mathrm{InAs} / \mathrm{AlSb} / \mathrm{InAs}$ the using nonequilibrium Green's function method. Kiledjian et al. ${ }^{11}$ calculated the $I-V$ characteristics of various InAs/AlSb/GaSb systems, and show the dependence of coupling of heavy-hole state on $k_{\|}$. Genoe et al. ${ }^{13}$ also calculated the relation of $k_{\|}$and the transmission probability of InAs/AlSb/GaSb/AlSb/InAs under eight-band theory. Yu et al. ${ }^{14}$ analyzed the $I$ - $V$ characteristics of InAs/GaSb/InAs with different GaSb layer thicknesses. Kitabayashi et al. ${ }^{15}$ investigated the $I-V$ characteristics of $\mathrm{InAs} / \mathrm{AlSb} / \mathrm{GaSb} / \mathrm{AlSb} / \mathrm{InAs}$ with extremely thin AlSb barriers, and found that when 0.5-ML-thick AlSb barrier layers were inserted, NDR appeared. González et al. ${ }^{16}$ measured the temperature dependence of zero-bias differential tunneling conductance of InAs/AlSb/GaSb/AlSb/InAs under a magnetic field.

So far, all the theoretical studies focused on dc transport. Since all devices are operated under an ac bias, it is important to study ac transport through interband structures. It has been emphasized by Buttiker ${ }^{17}$ that in dealing with ac transport, the current will not be conserved. This is because the expression of the current obtained either from the usual Landauer-Buttiker formula or the equation of motion using the nonequilibrium Green's function is for the conduction current. In the presence of ac bias, the displacement current due to the charge accumulation plays an important role and has to be considered. For this purpose, Büttiker et al. ${ }^{17}$ derived a general theory of the ac conductance for a multiprobe and multichannel conductor. The theory was formulated using the scattering matrix. In this paper, we numerically calculate the ac conductance of InAs/GaSb/InAs and InAs/ $\mathrm{AlSb} / \mathrm{GaSb} / \mathrm{AlSb} / \mathrm{InAs}$ structures using Buttiker's theory. A unique feature of the interband structure we considered is that it has an energy window of $\Delta E=0.15 \mathrm{eV}$ within which the transmission coefficient is nonzero. Because of this, we found that when the frequency of the external bias is comparable to the energy window, the real part of the dynamical conductance exhibit a series of plateaus. The number and width of the plateaus are equal to the number and level spacing of the resonant states in the interband structure, respectively. When the frequency is smaller than the energy window an extra plateau can appear, with the position depending on the frequency. At a frequency much smaller than $\Delta E$, the 
imaginary part of the dynamical conductance is well described by the emittance. ${ }^{18}$ As the frequency is increased, both the inductivelike behavior observed on the resonance and the capacitivelike behavior observed off the resonance are enhanced. The paper is organized as follows. In Sec. II, the theory for dynamical conductance and the numerical method are outlined. In Sec. III, the results are presented and discussed. The conclusion is given in Sec. IV.

\section{THEORY}

We start with the general expression for the ac conductance of a noninteracting system, ${ }^{17,19}$

$$
\begin{aligned}
G_{\alpha \beta}^{c}(\omega)= & \frac{e^{2}}{h} \int d E \operatorname{Tr}\left[1_{\alpha} \delta_{\alpha \beta}-s_{\alpha \beta}^{+}(E) s_{\alpha \beta}(E+\hbar \omega)\right] \\
& \times \frac{f(E)-f(E+\hbar \omega)}{\hbar \omega},
\end{aligned}
$$

where $s_{\alpha \beta}$ is the scattering matrix and $f(E)$ is the Fermi energy. At zero temperature, $f(E)$ is a step function and $f(E)=\theta\left(E-E_{F}\right)$. For a two probe system, we have (setting $e^{2} / h=1$ and $\left.\hbar=1\right)$

$$
G_{\alpha \beta}^{c}(\omega)=\frac{1}{\omega} \int_{E_{m}}^{E_{F}} d E\left[\delta_{\alpha \beta}-s_{\alpha \beta}^{*}(E) s_{\alpha \beta}(E+\omega)\right],
$$

with its real part and imaginary parts as

$$
\begin{aligned}
\operatorname{Re}\left[G_{\alpha \beta}^{c}(\omega)\right]= & \frac{1}{\omega} \int_{E_{m}}^{E_{F}}\left\{\delta_{\alpha \beta}-\operatorname{Re}\left[s_{\alpha \beta}^{*}(E)\right] \operatorname{Re}\left[s_{\alpha \beta}(E+\omega)\right]\right. \\
& \left.+\operatorname{Im}\left[s_{\alpha \beta}^{*}(E)\right] \operatorname{Im}\left[s_{\alpha \beta}(E+\omega)\right]\right\} d E \\
\operatorname{Im}\left[G_{\alpha \beta}^{c}(\omega)\right]= & \frac{-1}{\omega} \int_{E_{m}}^{E_{F}}\left\{\operatorname{Im}\left[s_{\alpha \beta}^{*}(E)\right] \operatorname{Re}\left[s_{\alpha \beta}(E+\omega)\right]\right. \\
& \left.+\operatorname{Re}\left[s_{\alpha \beta}^{*}(E)\right] \operatorname{Im}\left[s_{\alpha \beta}(E+\omega)\right]\right\} d E
\end{aligned}
$$

where $E_{m}=\max \left\{E_{F}-\omega, 0\right\}$ because no scattering state is allowed for negative energy. As pointed out by Buttiker, ${ }^{17} G_{11}^{c}$ is the contribution from the conduction current. The displacement current due to the charge accumulation has to be taken into account in order to conserve the current. To do that, one needs to include electron electron interaction at least to the Hartree level, and solve the Poisson equation. ${ }^{20}$ In this paper, we will use a phenomenological current conserving theory ${ }^{17,21}$ which includes the effect of electron electron interaction by the partition of the displacement current. The dynamical conductance of this theory is given by ${ }^{22}$

$$
\bar{G}_{\alpha \beta}(\omega)=G_{\alpha \beta}^{c}(\omega)-\frac{\sum_{\gamma} G_{\alpha \gamma}^{c}(\omega) \sum_{\delta} G_{\delta \beta}^{c}(\omega)}{\sum_{\gamma \delta} G_{\gamma \delta}^{c}(\omega)} .
$$

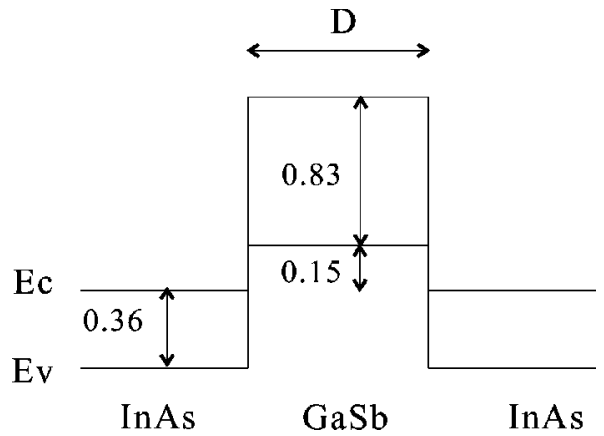

FIG. 1. The schematic band diagram of the InAs/GaSb/InAs structure.

The dynamical conductance $G=\bar{G}_{11}$ is a complex quantity, with its real part $G_{R}$ describing the dissipation and its imaginary part $G_{I}$ characterizing the phase difference between the current and the voltage. At small frequency, it is appropriate to describe $\bar{G}_{\alpha \beta}(\omega)$ using Buttiker's formalism ${ }^{18}$ according to which the dynamical conductance can be expanded as a power series in frequency $\omega, \bar{G}_{11}(\omega)=\bar{G}_{11}(0)-i \omega E_{11}$ $+\omega^{2} K_{11}+O\left(\omega^{3}\right)$. Here $E_{11}$ is the emittance ${ }^{18}$ which has been studied extensively. ${ }^{23}$ The emittance describes the response of the system to the external ac bias, and $K_{11}$ describes the correction to the real part of the dynamical conductance at small frequency. If $E_{11}$ is positive, the system responds like a capacitor. On the other hand, the system shows an inductivelike behavior for a negative emittance. ${ }^{24,25}$ Physically, the capacitivelike behavior means that the current follows the voltage and the inductivelike behavior means that the voltage follows the current. ${ }^{26}$

In this paper, we consider InAs/GaSb/InAs and InAs/ $\mathrm{AlSb} / \mathrm{GaSb} / \mathrm{AlSb} / \mathrm{InAs}$ structures. Based on the work of Yang and $\mathrm{Xu},{ }^{5} \Gamma$-point and flat-band approximations will be taken. Note that our system is an ideal model. The impurity scattering, emitter notch quantization, and band bending, as well as band mixing, have not been considered. Hence in the experiment, only qualitative features may be observed. The schematic band diagrams of InAs/AlSb/GaSb and InAs/AlSb/ $\mathrm{GaSb} / \mathrm{AlSb} / \mathrm{InAs}$ are shown in Figs. 1 and $2 .^{3-5}$ The band edge energy and the effective mass of the GaSb, AlSb, and InAs are taken as follows: ${ }^{5,27,28}$

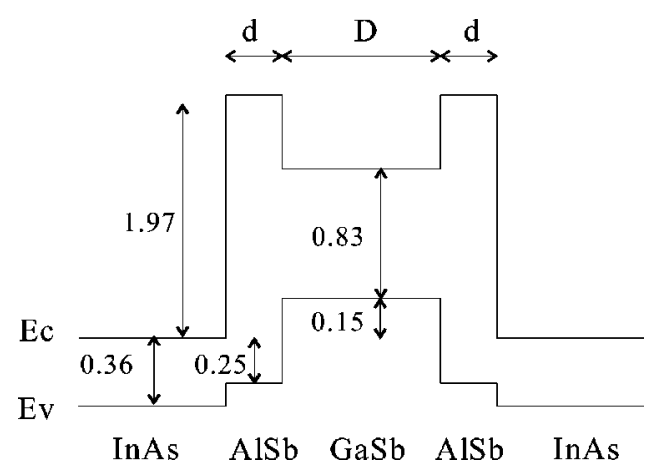

FIG. 2. The schematic band diagram of the InAs/AlSb/GaSb/ $\mathrm{AlSb} / \mathrm{InAs}$ structure. 


\begin{tabular}{lccc} 
& GaSb & AlSb & InAs \\
\hline \hline$E_{c}(\mathrm{eV})$ & 0.83 & 1.97 & 0 \\
$E_{v}(\mathrm{eV})$ & 0.15 & -0.25 & -0.36 \\
$m^{*} / m_{0}$ & 0.05 & 0.146 & 0.027
\end{tabular}

For this two-band model, the Hamiltonian is given by ${ }^{5}$

$$
H=\left(\begin{array}{cc}
E_{c}+\frac{\hbar^{2}}{2 m_{0}} k^{2} & \hbar P k \\
\hbar P k & E_{v}-\frac{\hbar^{2}}{2 m_{0}} k^{2}
\end{array}\right),
$$

where $P$ is a constant. In order to calculate the dynamical conductance, we have to obtain the scattering matrix first. For the InAs/GaSb/InAs structure, the scattering matrix can be solved exactly,

$$
s_{11}=\frac{\beta_{k_{1}}^{2}+\beta_{k_{2}^{\prime}}^{2}}{F}\left(e^{2 k_{2}^{\prime} L}-1\right)
$$

and

$$
s_{21}=\frac{4 i \beta_{k_{1}} \beta_{k_{2}^{\prime}}}{F} e^{k_{2}^{\prime} L},
$$

where

$$
F=\left(-1+e^{2 k_{2}^{\prime} L}\right)\left(\beta_{k_{1}}^{2}-\beta_{k_{2}^{\prime}}^{2}\right)+2 i\left(1+e^{2 k_{2}^{\prime} L}\right) \beta_{k_{1}} \beta_{k_{2}^{\prime}},
$$

and $\left(k_{1}, \beta_{k_{1}}\right)$ and $\left(i k_{2}^{\prime}, i \beta_{k_{2}^{\prime}}\right)$ are the $\left(k, \beta_{k}\right)$ values of the InAs and GaSb layers, respectively. Here $L$ is the thickness of the GaSb layer, and

$$
\begin{gathered}
\beta_{k}=\hbar P k /\left(E-E_{v}\right), \\
k=\left[\left(E-E_{c}\right)\left(E-E_{v}\right)\right]^{1 / 2} /(\hbar P) .
\end{gathered}
$$

We can calculate the value of $P$ by using the following equation:

$$
m^{*}=\left[m_{0}^{-1}+2 P^{2} /\left(E_{c}-E_{v}\right)\right]^{-1} .
$$

When $L$ is large, $e^{k_{2}^{\prime} L} \gg 1$, the term $e^{2 k_{2}^{\prime} L}$ dominant, and so,

$$
s_{11} \simeq \frac{\beta_{k_{1}}^{2}-\beta_{k_{2}^{\prime}}^{2}}{\beta_{k_{1}}^{2}+\beta_{k_{2}^{\prime}}^{2}}-\frac{2 i \beta_{k_{1}} \beta_{k_{2}^{\prime}}}{\beta_{k_{1}}^{2}+\beta_{k_{2}^{\prime}}^{2}}
$$

which shows that when $L$ is large the $s_{11}$ is approximately independent of $L$.

For the InAs/AlSb/GaSb/AlSb/InAs structures, the scattering matrix can be easily calculated numerically. In this paper, we use the one-dimensional transfer matrix method ${ }^{4}$ which is suitable for the two-band $\mathrm{k} \cdot \mathrm{p}$ model. The solution of the two-band Schrödinger equation is ${ }^{29}$

$$
\left[\begin{array}{c}
\Psi_{c} \\
\Psi_{v}
\end{array}\right]=\left[\begin{array}{c}
A \exp (i k z)+B \exp (-i k z) \\
\beta_{k}[A \exp (i k z)-B \exp (-i k z)]
\end{array}\right],
$$

where $A$ and $B$ are coefficients of each layer. By matching wave functions at the boundary of interface and multiplying the transfer matrix of all interfaces, we obtain a $2 \times 2$ transfer matrix $M$ with a matrix element $m_{i, j}$,

$$
\left[\begin{array}{l}
A_{1} \\
B_{1}
\end{array}\right]=M\left[\begin{array}{l}
A_{n} \\
B_{n}
\end{array}\right]
$$

where $A_{1}, B_{1}$ and $A_{n}, B_{n}$ are the coefficients of the first and last layers, respectively. Then, the scattering matrix is defined as

$$
\left[\begin{array}{c}
B_{1} \\
A_{n} \exp (i k L)
\end{array}\right]=S\left[\begin{array}{c}
A_{1} \\
B_{n} \exp (-i k L)
\end{array}\right]
$$

from which we obtain

$$
S=\left(\begin{array}{cc}
m_{21} / m_{11} & \left(m_{22}-m_{12} m_{21} / m_{11}\right) \exp (i k L) \\
\exp (i k L) / m_{11} & -m_{12} \exp (2 i k L) / m_{11}
\end{array}\right),
$$

where $L=D+2 d, D$ is the thickness of the GaSb layer, and $d$ is the thickness of the AlSb layer. Equations (3), (4), and (17) allow us to obtain the dynamical conductance numerically.

\section{NUMERICAL RESULTS AND DISCUSSION}

The numerical results of $\bar{G}_{11}$ versus the Fermi energy $E_{F}$ at different frequencies $\omega$ and different thicknesses of layers are calculated using Eqs. (3) and (4). In all of the plots the unit of conductance is measured in $e^{2} / h$ and the frequency is measured in $\mathrm{eV}$.

Figure 3 shows the real and imaginary parts of $\bar{G}_{11}$ versus $E_{F}$ at small frequencies with $D=300 \AA$ and $d=0$. For $D$ $=300 \AA$ and $d=0$, four resonant states are found in the energy window ranging from $E_{c}$ of InAs to $E_{c}+0.15 \mathrm{eV}$. Different from the normal case (the one-band model) where the higher resonant level has a broader peak, for the interband model (two-band model) studied here the situation is reversed. When the frequency is turned on with $\omega$ $=0.001 \mathrm{eV}$, the conductance at different energies are mixed. As a result, the resonance is suppressed. This suppression of the resonant peak is most significant for the sharpest peak at higher energy. As one increases the frequency to $\omega=0.005$ or $0.01 \mathrm{eV}$, the peak value of the resonance drops drastically and a double-peak structure shows up. This behavior can be understood as follows. Near a resonant peak, the scattering matrix can be described by the Breit-Wigner formula. For a symmetric system, we have

$$
s_{\alpha \beta}=\delta_{\alpha \beta}-i \frac{\Gamma / 2}{\Delta E+i \Gamma / 2},
$$

where $\Delta E=E-E_{r}, E_{r}$ is the resonant level, and $\Gamma / 2$ describes the lift time of the resonant level which also characterizes the half-width of the resonant peak. If the scattering matrix can be approximated by its Breit-Wigner form, the dynamical conductance can be obtained exactly, ${ }^{24,21}$ 

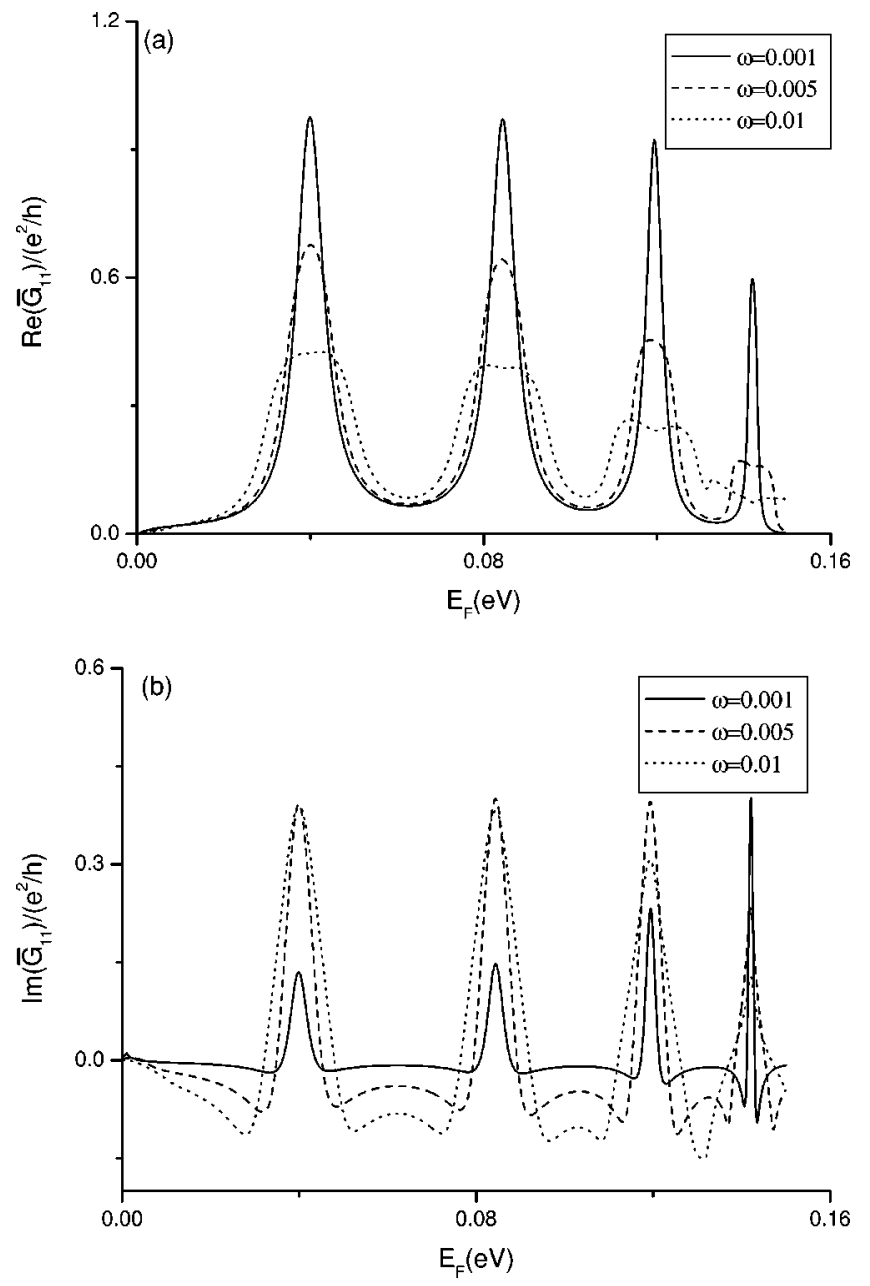

FIG. 3. Dynamical conductance vs Fermi energy $E_{F}$ at small frequencies. (a) Real part: $\operatorname{Re}\left(\bar{G}_{11}\right)$. (b) Imaginary part: $\operatorname{Im}\left(\bar{G}_{11}\right)$. Here $D=300 \AA$ and $d=0$.

$$
\operatorname{Re}\left[G_{11}^{c}\right]=\frac{\Gamma}{8 \pi \omega}\left\{\arctan \left[\frac{\Delta E+\omega}{\Gamma / 2}\right]-\arctan \left[\frac{\Delta E-\omega}{\Gamma / 2}\right]\right\}
$$

and

$$
\begin{aligned}
\operatorname{Im}\left[G_{11}^{c}\right]= & \frac{\Gamma}{16 \pi \omega} \ln \left\{[ ( \Delta E + \omega ) ^ { 2 } + ( \Gamma / 2 ) ^ { 2 } ] \left[(\Delta E-\omega)^{2}\right.\right. \\
& \left.\left.+(\Gamma / 2)^{2}\right]\left[(\Delta E)^{2}+(\Gamma / 2)^{2}\right]^{-2}\right\}
\end{aligned}
$$

For small $\omega$ and near a resonance, the emittance and the quantity $K_{11}$ has been obtained from the Breit-Wigner formulas [Eqs. (19) and (20)] (Refs. 26 and 30)

$$
E_{11}=-\frac{\Gamma}{8 \pi} \frac{\Gamma^{2} / 2-\Delta^{2}}{\Delta^{4}}
$$

and

$$
K_{11}=\frac{\pi^{2}}{\Gamma}\left[\left(E-E_{r}\right)^{2}-\Gamma^{2} / 12\right]\left(\frac{d N}{d E}\right)^{3},
$$

where the density of states (DOS) $d N / d E$ is defined as

$$
\frac{d N}{d E}=\frac{1}{2 \pi} \frac{\Gamma}{\Delta^{2}}
$$

with $\Delta^{2}=\left(E-E_{r}\right)^{2}+\Gamma^{2} / 4$. Following observations are in order. First, at the resonance, $E_{11} \sim(\omega / \Gamma)$ and $K_{11}$ $\sim(\omega / \Gamma)^{2}$. Second, we see that at the resonance the emittance is positive and becomes negative when $\left|E-E_{r}\right|$ $>\Gamma / 2$. Furthermore, near the resonance $G_{R}(\omega)<G_{R}(0)$ because $K_{11}<0$. However, when $\left|E-E_{r}\right|>\Gamma / 2 \sqrt{3}, K_{11}$ becomes positive. Thus on the resonance, the real part of the dynamical conductance $\operatorname{Re}\left[\bar{G}_{11}\right]$ is always smaller than the corresponding dc conductance. Since the DOS $d N / d E$ $\sim \Gamma^{-1}$ at the resonance, we find $K_{11} \sim-\Gamma^{-2}$. Thus, a larger reduction of $\operatorname{Re}\left(\bar{G}_{11}\right)$ for a sharper peak (with a smaller $\Gamma$ ) as is the case for the fourth peak in Fig. 3(a). Equation (22) also shows a double-peak structure with two maxima at $E$ $=E_{r} \pm \Gamma / 2$ which agrees what was shown in Fig. 3(a). We now examine the imaginary part of the dynamical conductance $\operatorname{Im}\left[\bar{G}_{11}\right]$ [Fig. 3(b)]. Generally speaking, the inductivelike behavior on the resonance and the capacitivelike behavior off the resonance are enhanced. Specifically, at a small frequency $\omega=0.001 \mathrm{eV}$, we see a typical behavior of the emittance of resonant peaks governed by Eq. (21). According to Eq. (21), the smaller half-width $\Gamma$ gives rise to a larger peak value for the emittance. When the frequency is increased to $\omega=0.005 \mathrm{eV}$, the peak value of $\operatorname{Im}\left[\bar{G}_{11}\right]$ for the first three resonant peaks increases according to Eq. (21) due to their relatively large $\Gamma$ compared with $\omega=0.005 \mathrm{eV}$. The behaviors of $\operatorname{Re}\left[\bar{G}_{11}\right]$ and $\operatorname{Im}\left[\bar{G}_{11}\right]$ at small $\omega$ can also be understood qualitatively from a classical circuit model. ${ }^{30}$ Due to both inductivelike and capacitivelike responses, our system can be considered as an inductor in series with a parallel connection of a capacitor and a resistor. For this classical circuit the dynamic conductance can be written ${ }^{30}$ in the following form up to the second order in frequency $\omega$ :

$$
G(\omega)=\frac{1}{R}-i \omega\left[C-\frac{L}{R^{2}}\right]+\omega^{2} \frac{L}{R}\left[2 C-\frac{L}{R^{2}}\right] .
$$

The linear term in $\omega$, which corresponds to the nondissipative part of the dynamic conductance $\operatorname{Im}\left[\bar{G}_{11}\right]$, exhibits a competition between two different dynamic responses. If $C$ $>L / R^{2}$, the response is capacitivelike with a negative $\operatorname{Im}\left[\bar{G}_{11}\right]$, and $\left|\operatorname{Im}\left[\bar{G}_{11}\right]\right|$ increases linearly with $\omega$ at small frequencies, which is consistent with our calculation. A similar argument for $\operatorname{Im}\left[\bar{G}_{11}\right]$ applies to the inductivelike region when $C<L / R^{2}$. The transition from capacitivelike to inductivelike behavior occurs when $C=L / R^{2}$, when $\operatorname{Im}\left[\bar{G}_{11}\right]$ vanishes to the second order in $\omega$. On the other hand, the dissipative component $\operatorname{Re}\left[\bar{G}_{11}\right]$, near the resonant point, can increase or decrease with $\omega^{2}$ depending on the sign of the second-order term in Eq. (24). According to this picture $\operatorname{Re}\left[\bar{G}_{11}\right]$ decreases when $C<L /\left(2 R^{2}\right)$ and increases otherwise, which is consistent with Fig. 3(a). Note, however, that when the frequency is so large that $\omega^{3}$ or higher order terms cannot be neglected, Eq. (24) breaks down. 

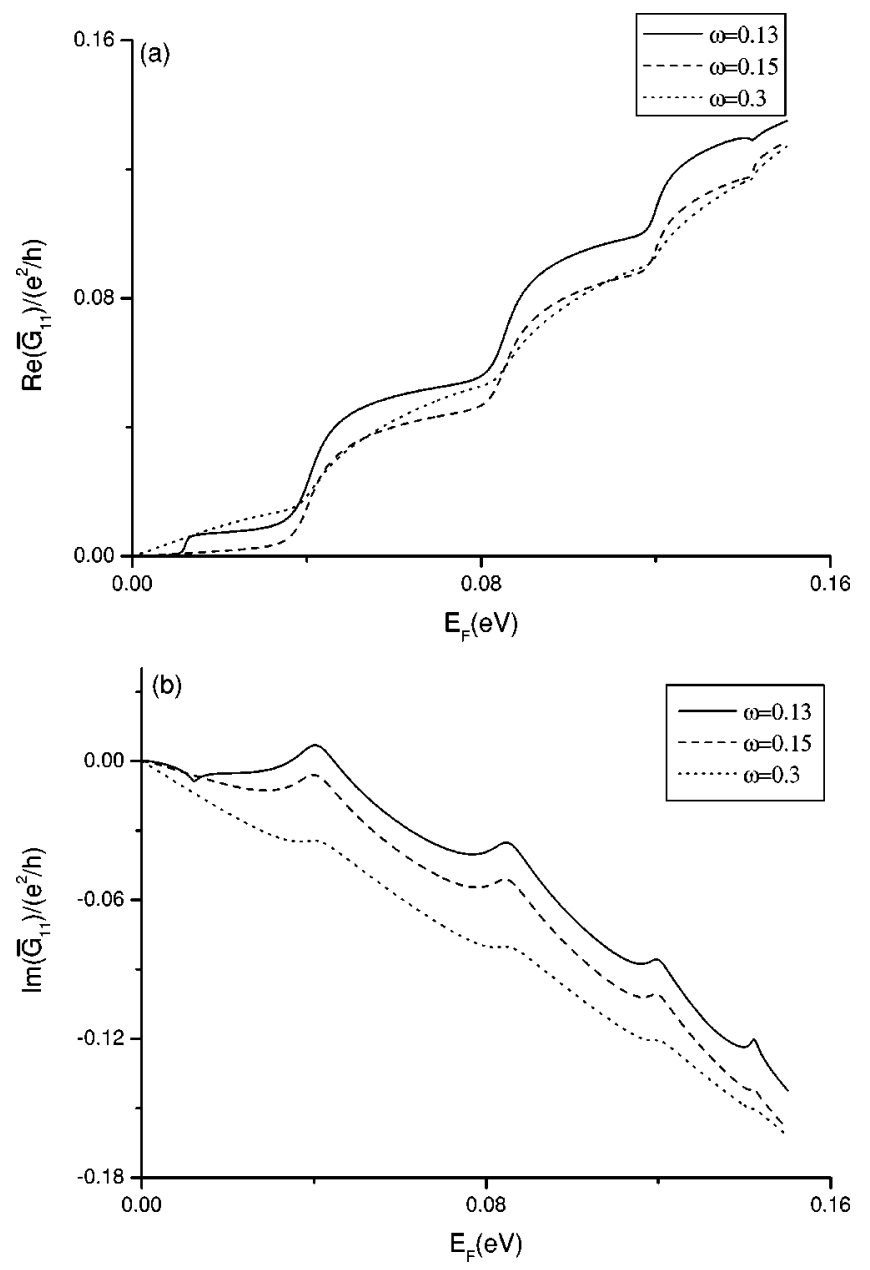

FIG. 4. Dynamical conductance vs Fermi energy $E_{F}$ at large frequencies. (a) Real part: $\operatorname{Re}\left(\bar{G}_{11}\right)$. (b) Imaginary part: $\operatorname{Im}\left(\bar{G}_{11}\right)$. Here $D=300 \AA$ and $d=0$.

When $\omega$ is increased further to $0.01 \mathrm{eV}$, the higher order terms in the expansion of the dynamical conductance have to be considered. Thus, the peak value of $\operatorname{Im}\left[\bar{G}_{11}\right]$ saturate for the first and second resonant peaks and decreases for the third peak. Since the half-width of the fourth resonant peak is smaller than $\omega=0.005 \mathrm{eV}, \operatorname{Im}\left[\bar{G}_{11}\right]$ decreases quickly as the frequency is increased according to Eq. (20). When the frequency is further increased by one order of magnitude to $\omega$ $=0.13$ and $0.3 \mathrm{eV}$, which are comparable to the transmission window $(\Delta E=0.15 \mathrm{eV})$ of the interband structure, we see plateaulike structures for the real part of dynamical conductance [Fig. 4(a)]. This plateaulike structure and the resonant peak structure in Fig. 3(a) (solid line) are well correlated with resonant peak positions located at the start of each plateau. The physics behind it can be explained as follows. From Eq. (3), we see that $\operatorname{Re}\left(G_{11}^{c}\right)$ consists of three terms. The steplike plateaus come from the first two terms in Eq. (3). When $\omega \sim 0.15 \mathrm{eV}$ the integration ranges in Eqs. (3) and (4) start from zero. This is because the transmission energy window is $0.15 \mathrm{eV}$, i.e., the range of $E_{F}$ is $0.15 \mathrm{eV}$. There is no transmissive state when $E<0$. When $E$ is between 0.15 and $0.83 \mathrm{eV}$, the transmission coefficient is almost zero due

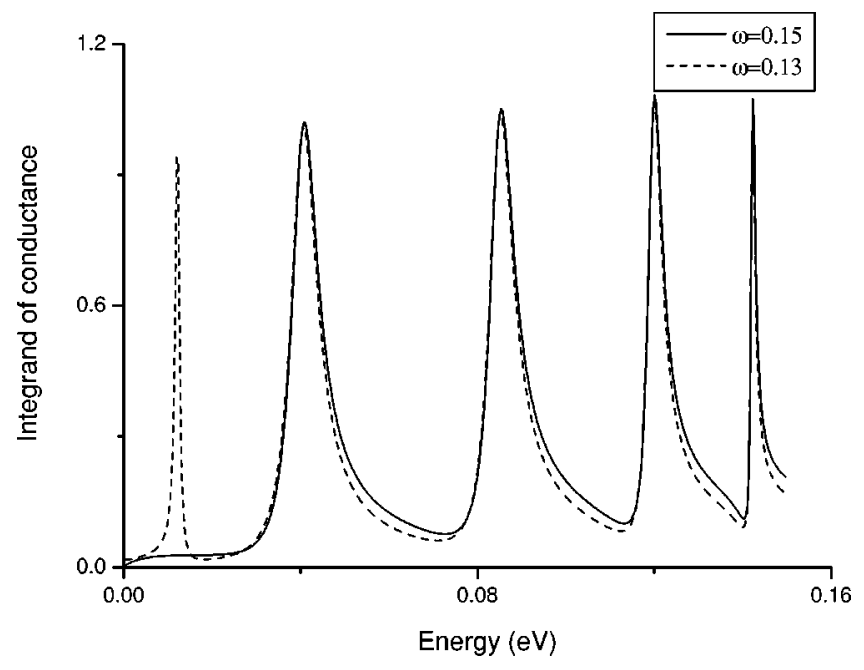

FIG. 5. The integrand of Eq. (3) vs energy $E$ at different frequencies. Here $D=300 \AA$ and $d=0$.

to the large barrier of GaSb. When $\omega=0.15 \mathrm{eV}, s_{11}(E+\omega)$ is roughly a slow varying function of $E$ whereas $\operatorname{Re}\left[s_{11}(E)\right]$ has a resonant feature with peaks at resonant levels. ${ }^{31}$ So the second term of Eq. (3) has a similar behavior of $\operatorname{Re}\left[s_{11}(E)\right]$. As a result, the integrand of Eq. (3) exhibits peaks at resonant levels very similar to the transmission coefficient (see the solid line in Fig. 5). After integration from $E=0$ to $E$ $=E_{F}$, the conductance jumps whenever $E_{F}$ hits a resonant peak giving rise to the plateaulike structure. The third term of Eq. (3) is much small but not negligible and it rounds off the sharp plateaus. For $\omega=0.3 \mathrm{eV}$ we see a similar behavior, but the plateau is not as flat. For $\omega=0.13 \mathrm{eV}$, we see an additional plateau. This can also be understood within the picture discussed above. When $\omega=0.13 \mathrm{eV}, \operatorname{Re}\left[s_{11}(E\right.$ $+\omega)]$ is a slowly varying function of $E$ when $E>0.02 \mathrm{eV}$. However, when $E<0.02 \mathrm{eV}, \operatorname{Re}\left[s_{11}(E+\omega)\right]$ contains the resonant feature of the fourth peak in the transmission coefficient at $E \sim 0.01 \mathrm{eV}$. The product of $\operatorname{Re}\left[s_{11}(E+\omega)\right]$ and $\operatorname{Re}\left[s_{11}(E)\right]$ gives rise an extra peak at $E=0.01 \mathrm{eV}$ in addition to the case of $\omega=0.15 \mathrm{eV}$ (see the dashed line in Fig. 5). This extra peak in turn gives rise to the extra plateau in Fig. 4 (a) (solid line). Moreover, when $\omega<0.1$, the curve is rather complicated and irregular. For the imaginary part of the conductance, we see a spikelike behavior which can also be interpreted using the above picture. Now we discuss the influence of the thickness of the barriers inserted in the structure (Fig. 6). As barriers are put in, this further confinement pushes the resonant states up. ${ }^{32}$ As a result, we see that the positions of the plateaus are shifted to the right. Moreover, the height of the plateaus are lower and flatter than before. Note that there is an extra plateau when the barriers are inserted. This is because one more resonant state emerges within the energy window. Similarly, the spikes of the imaginary part of the dynamical conductance also move to the high energy end with the appearance of an additional spike. Finally, in Fig. 7 we doubled the width of the GaSb layer $D$. Approximately, the number of resonant states are also doubled, and so are the number of plateaus (spikes) in the real (imaginary) part of the dynamical conductance. 

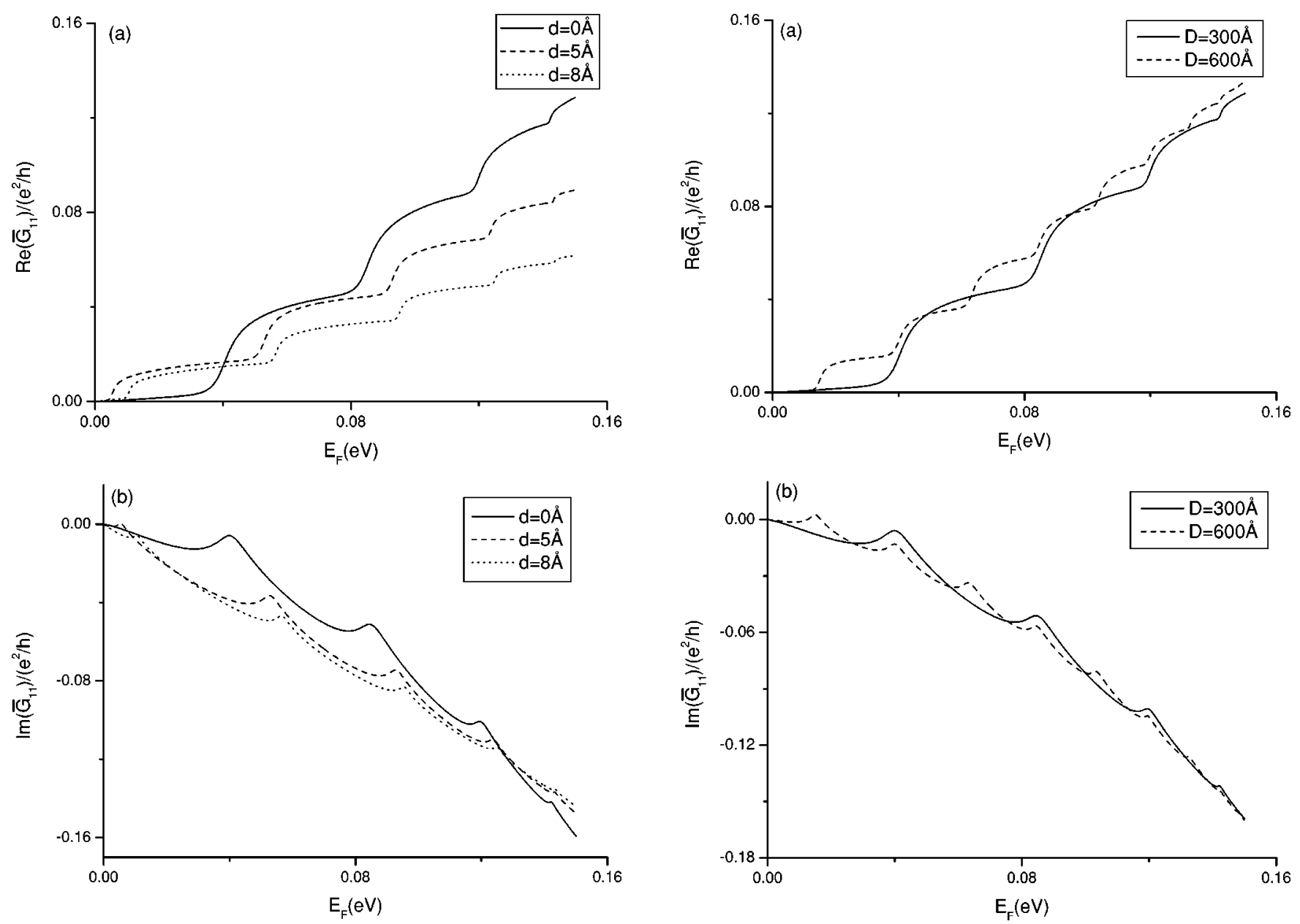

FIG. 6. Dynamical conductance vs Fermi energy $E_{F}$ at different barrier widths. (a) Real part: $\operatorname{Re}\left(\bar{G}_{11}\right)$. (b) Imaginary part: $\operatorname{Im}\left(\bar{G}_{11}\right)$. Here $D=300 \AA$ and $\omega=0.15 \mathrm{eV}$.

\section{CONCLUSION}

Using current conserving and gauge invariant ac transport theory, numerical solutions of the dynamical conductance $\bar{G}_{11}$ are obtained for InAs/GaSb/InAs and InAs/AlSb/GaSb/ $\mathrm{AlSb} / \mathrm{InAs}$ structures. In these structures, there exists an energy window $\Delta E=0.15 \mathrm{eV}$ such that the transmission coefficient is zero when the Fermi energy is outside of the window. When the frequency of the external bias is smaller than the lifetime of the resonant state, the response of the system is well described by the emittance. Generally speaking, the inductivelike (capacitive-like) behavior when the system on (off) the resonance is enhanced as the frequency is increased. When the frequency is comparable to the energy window $\Delta E$, the real part of the dynamical conductance ex-

FIG. 7. Dynamical conductance vs Fermi energy $E_{F}$ at different D. (a) Real part: $\operatorname{Re}\left(\bar{G}_{11}\right)$. (b) Imaginary part: $\operatorname{Im}\left(\bar{G}_{11}\right)$. Here $d$ $=0$ and $\omega=0.15 \mathrm{eV}$.

hibits a series of plateaus which is well correlated with the profile of the transmission coefficient. We found that the widths of the plateaus are equal to the level spacings of the resonant states. The numbers and positions of the plateaus can be controlled by changing the external frequency. This unique property for the interband structure may have potential device applications. A spikelike structure is also found for the imaginary part of the dynamical conductance within this frequency range.

\section{ACKNOWLEDGMENT}

We gratefully acknowledge financial support from RGC grant (HKU 7113/02P) from the government of Hong Kong SAR and a grant from CRCG of The University of Hong Kong.

*Electronic address: jianwang@hkusub.hku.hk

${ }^{1}$ L. Esaki, L.L. Chang, and E.E. Mendez, Jpn. J. Appl. Phys. 20, L529 (1981).

${ }^{2}$ M. Sweeny and J.M. Xu, Appl. Phys. Lett. 54, 546 (1989).

${ }^{3}$ J.R. Söderström, D.H. Chow, and T.C. McGill, Appl. Phys. Lett.
55, 1094 (1989).

${ }^{4}$ R.Q. Yang and J.M. Xu, Phys. Rev. B 46, 6969 (1992).

${ }^{5}$ R.Q. Yang and J.M. Xu, J. Appl. Phys. 72, 4714 (1992).

${ }^{6}$ J. Wang, Y.J. Wang, and H. Guo, J. Appl. Phys. 75, 2724 (1994).

${ }^{7}$ E.O. Kane, in Semiconductors and Semimetals, edited by R.K. 
Willardson and A.C. Beer (Academic, New York, 1996), Vol. 1, p. 75.

${ }^{8}$ D.Z.-Y. Ting, E.T. Yu, and T.C. McGill, Appl. Phys. Lett. 58, 292 (1991).

${ }^{9}$ C. Aversa and J.E. Sipe, Appl. Phys. Lett. 63, 1975 (1993).

${ }^{10}$ S.R. White and L.J. Sham, Phys. Rev. Lett. 47, 879 (1981).

${ }^{11}$ M.S. Kiledjian, J.N. Schulman, K.L. Wang, and K.V. Rousseau, Phys. Rev. B 46, 16012 (1992).

${ }^{12}$ M.A. Davidovich, E.V. Anda, C. Tejedor, and G. Platero, Phys. Rev. B 47, 4475 (1993).

${ }^{13}$ J. Genoe, K. Fobelets, C. Van Hoof, and G. Borghs, Phys. Rev. B 52, 14025 (1995).

${ }^{14}$ E.T. Yu, D.A. Collins, D.Z.-Y. Ting, D.H. Chow, and T.C. McGill, Appl. Phys. Lett. 57, 2675 (1990).

${ }^{15}$ H. Kitabayshi, T. Waho, and M. Yamamoto, Appl. Phys. Lett. 71, 512 (1997).

${ }^{16}$ E.M. González, Y. Lin, and E.E. Mendez, Phys. Rev. B 63, 033308 (2001).

${ }^{17}$ M. Büttiker, A. Prêtre, and H. Thomas, Phys. Rev. Lett. 70, 4114 (1993).

${ }^{18}$ M. Büttiker, J. Phys.: Condens. Matter 5, 9361 (1993).

${ }^{19}$ In this paper, we only consider the one-dimensional system. For a three-dimensional layered structure, the momentum parallel to the plane of the three-dimensional layer has to be considered. Then Eq. (1) has to be modified. The conductance density $\mathcal{G}^{c}$ should be

$$
\begin{aligned}
\mathcal{G}_{\alpha \beta}^{c}(\omega)= & \frac{e^{2} m^{*} k T}{4 \pi^{2} \hbar^{4} \omega} \int d E \operatorname{Tr}\left[1_{\alpha} \delta_{\alpha \beta}-s_{\alpha \beta}^{+}(E) s_{\alpha \beta}(E\right. \\
& +\hbar \omega)] \log \left[\frac{1+\exp \left[\left(E_{F}-E\right) / k T\right]}{1+\exp \left[\left(E_{F}-E-\hbar \omega\right) / k T\right]}\right] .
\end{aligned}
$$

${ }^{20}$ M. Büttiker and T. Christen, in Quantum Transport in Semiconductor Submicron Structures, edited by B. Kramer (Kluwer,
Dordrecht, 1996), p. 263; Z.S. Ma, J. Wang, and H. Guo, Phys. Rev. B 57, 9108 (1998); 59, 7575 (1999).

${ }^{21}$ B.G. Wang, J. Wang, and H. Guo, Phys. Rev. Lett. 82, 398 (1999).

${ }^{22}$ Equation (16) of Ref. 17 is for a three-lead system: two conducting leads and a third lead capacitively coupled to the system. If the third lead is absent, i.e., taking the limit when capacitance $C \rightarrow 0$, it reduces to Eq. (5).

${ }^{23}$ T. Christen and M. Buttiker, Phys. Rev. B 53, 2064 (1996); J. Wang and H. Guo, ibid. 54, R11 090 (1996); Q.R. Zheng, J. Wang, and H. Guo, ibid. 56, 12462 (1997); C.C. Wan, J.L. Mozos, J. Wang, and H. Guo, ibid. 55, R13 393 (1997); W.D. Sheng, Q.R. Zheng, J. Wang, and H. Guo, ibid. 59, 538 (1999); T. Gramespacher and M. Buttiker, ibid. 60, 2375 (1999); T. De Jesus, H. Guo, and J. Wang, ibid. 62, 10774 (2000); C. Roland, M.B. Nardelli, J. Wang, and H. Guo, Phys. Rev. Lett. 84, 2921 (2000).

${ }^{24}$ M. Buttiker and T. Christen, in Mesoscopic Electron Transport, edited by L.L. Sohn et al. (Kluwer, Dordrecht, 1997), p. 259.

${ }^{25}$ W. Zheng, Y.D. Wei, J. Wang, and H. Guo, Phys. Rev. B 61, 13121 (2000).

${ }^{26}$ M. Buttiker, H. Thomas, and A. Pretre, Z. Phys. B: Condens. Matter 94, 133 (1994).

${ }^{27}$ I.M. Tsidilkovski, Band Structure of Semiconductor (Pergamon, New York, 1982).

${ }^{28}$ A. Sasaki, Phys. Rev. B 30, 7016 (1984).

${ }^{29}$ Here we have neglected the kinetic energy which is small as pointed out in Ref. 10.

${ }^{30}$ A. Pretre, H. Thomas, and M. Buttiker, Phys. Rev. B 54, 8130 (1996).

${ }^{31}$ Note that $\left|s_{11}\right|$ has dips at resonant energies whereas $\operatorname{Re}\left(s_{11}\right)$ has peaks at the resonance.

${ }^{32}$ J. Wang and H. Guo, Appl. Phys. Lett. 60, 654 (1992). 